\title{
Accuracy of Capsule Colonoscopy in Detecting Colorectal Polyps in a Screening Population
}

\author{
Douglas K. Rex, ${ }^{1}$ Samuel N. Adler, ${ }^{2}$ James Aisenberg, ${ }^{3}$ Wilmot C. Burch Jr, ${ }^{4}$ \\ Cristina Carretero, ${ }^{5}$ Yehuda Chowers, ${ }^{6}$ Steven A. Fein, ${ }^{7}$ Steven E. Fern, ${ }^{8}$ \\ Ignacio Fernandez-Urien Sainz, ${ }^{9}$ Alexander Fich, ${ }^{10}$ Eyal Gal, ${ }^{11}$ John C. Horlander Sr, ${ }^{12}$ \\ Kim L. Isaacs, ${ }^{13}$ Revital Kariv, ${ }^{14}$ Adi Lahat, ${ }^{15}$ Wai-Keung Leung, ${ }^{16}$ Pramod R. Malik, ${ }^{17}$ \\ Doug Morgan, ${ }^{18}$ Neofytos Papageorgiou, ${ }^{19}$ David P. Romeo, ${ }^{20}$ Smita S. Shah, ${ }^{21}$ and \\ Matti Waterman ${ }^{6}$
}

${ }^{1}$ Department of Medicine, Division of Gastroenterology/Hepatology, Indiana University Hospital, Indianapolis, Indiana; ${ }^{2}$ Digestive Diseases Institute, Shaare Zedek Medical Center, Jerusalem, Israel; ${ }^{3}$ Division of Gastroenterology, Icahn School of Medicine at Mount Sinai, New York, New York; ${ }^{4}$ Franklin Gastroenterology, PLLC, Franklin, Tennessee; ${ }^{5}$ Departamento de Digestivo Clinica Universidad de Navarra, Pamplona, Spain; ${ }^{6}$ Division of Internal Medicine, Gastroenterology Institute, Rambam Health Care Campus, Haifa, Israel; ${ }^{7}$ Pasadena Gastroenterology Associates, PA, Pasadena, Texas; ${ }^{8}$ Specialists in Gastroenterology, Creve Coeur, Missouri; ${ }^{9}$ Department of Gastroenterology, Servicio de Digestivo, Hospital de Navarra, Pamplona, Spain; ${ }^{10}$ Department of Gastroenterology and Hepatology, Soroka University Medical Center, Beer-Sheva, Israel; ${ }^{11}$ Gastroenterology Department, Rabin Medical Center, Beilinson Hospital, Petah-Tikva, Israel; ${ }^{12}$ Louisville Gastroenterology Associates, PLLC, Lousiville, Kentucky; ${ }^{13}$ Department of Medicine, Division of Gastroenterology and Hepatology, University of North Carolina, Chapel Hill, North Carolina; ${ }^{14}$ The Research Center for Digestive Tract and Liver Diseases, The Tel-Aviv Sourasky Medical Center, Tel-Aviv, Israel; ${ }^{15}$ Department of Gastroenterology, Chaim Sheba Medical Center, Tel-Hashomer, Israel; ${ }^{16}$ Department of Medicine, Division of Gastroenterology \& Hepatology, Department of Medicine, University of Hong Kong, Hong Kong, China; ${ }^{17}$ Gastroenterology Associates of Tidewater, PC, Chesapeake, Virginia; ${ }^{18}$ Department of Medicine, Division of Gastroenterology, Hepatology, and Nutrition, Vanderbilt Institute for Global Health, Vanderbilt University, Nashville, Tennessee; ${ }^{19}$ Department of Gastroenterology and Hepatology, Sheba Medical Center Tel Hashomer, Ramat Gan, Israel; ${ }^{20}$ Dayton Gastroenterology, Inc, Beavercreek, Ohio; ${ }^{21}$ Alabama Digestive Disorders Center, PC, Huntsville, Alabama

See Covering the Cover synopsis on page 865; see editorial on page 892 .

BACKGROUND \& AIMS: Capsule colonoscopy is a minimally invasive imaging method. We measured the accuracy of this technology in detecting polyps $6 \mathrm{~mm}$ or larger in an averagerisk screening population. METHODS: In a prospective study, asymptomatic subjects $(\mathrm{n}=884)$ underwent capsule colonoscopy followed by conventional colonoscopy (the reference) several weeks later, with an endoscopist blinded to capsule results, at 10 centers in the United States and 6 centers in Israel from June 2011 through April 2012. An unblinded colonoscopy was performed on subjects found to have lesions $6 \mathrm{~mm}$ or larger by capsule but not conventional colonoscopy. RESULTS: Among the 884 subjects enrolled, 695 (79\%) were included in the analysis of capsule performance for all polyps. There were 77 exclusions (9\%) for inadequate cleansing and whole-colon capsule transit time fewer than 40 minutes, 45 exclusions (5\%) before capsule ingestion, 15 exclusions (2\%) after ingestion and before colonoscopy, and 15 exclusions $(2 \%)$ for site termination. Capsule colonoscopy identified subjects with 1 or more polyps $6 \mathrm{~mm}$ or larger with $81 \%$ sensitivity (95\% confidence interval [CI], 77\%-84\%) and 93\% specificity (95\% CI, 91\%-95\%), and polyps $10 \mathrm{~mm}$ or larger with $80 \%$ sensitivity (95\% CI, 74\%-86\%) and 97\% specificity (95\% CI, 96\%98\%). Capsule colonoscopy identified subjects with 1 or more conventional adenomas $6 \mathrm{~mm}$ or larger with $88 \%$ sensitivity (95\% CI, 82\%-93) and $82 \%$ specificity (95\% CI, 80\%-83\%), and $10 \mathrm{~mm}$ or larger with $92 \%$ sensitivity (95\% CI, 82\%-97\%) and 95\% specificity (95\% CI, 94\%-95\%). Sessile serrated polyps and hyperplastic polyps accounted for $26 \%$ and $37 \%$, respectively, of false-negative findings from capsule analyses. CONCLUSIONS: In an average-risk screening population, technically adequate capsule colonoscopy identified individuals with 1 or more conventional adenomas $6 \mathrm{~mm}$ or larger with $88 \%$ sensitivity and $82 \%$ specificity. Capsule performance seems adequate for patients who cannot undergo colonoscopy or who had incomplete colonoscopies. Additional studies are needed to improve capsule detection of serrated lesions. Clinicaltrials.gov number: NCT01372878.

Keywords: Colon Cancer Detection; Diagnosis; Tumor; Colorectal Lesion; Neoplasm.

Capsule endoscopy is valuable for investigation of small-bowel disease, but adaptation of capsule technology to colorectal imaging is challenging. A firstgeneration colon capsule produced variable results, however, one influential trial showed only $74 \%$ sensitivity for cancer. ${ }^{1}$ The first-generation capsule captured photographs at a constant 4 frames per second. The second-generation

Abbreviations used in this paper: ADR, adenoma detection rate; $\mathrm{Cl}$, confidence interval; CRC, colorectal cancer; CT, computed tomography; CTC, computed tomography colonography. 
capsule features motion detection and variable frame speed, with photographs at 4 frames per second when stationary and 35 frames per second when moving., ${ }^{2,3}$ The angle of view of the imagers increased from $156^{\circ}$ to $172^{\circ}$ from first to second generation.

Two studies, each including approximately 100 patients, found second-generation capsule sensitivities of $89 \%$ and $84 \%$ for detecting patients with polyps $6 \mathrm{~mm}$ or larger (specificity, $76 \%$ and $64 \%$, respectively). ${ }^{2,3}$ For patients with polyps $10 \mathrm{~mm}$ or larger in size, the sensitivities were $88 \%$ in both studies, with specificities of $89 \%$ and $95 \%$. $^{2,3}$

The current study evaluated the second-generation capsule for detecting patients with polyps $6 \mathrm{~mm}$ or larger in an average-risk screening population. The study was conducted in 16 centers in the United States and Israel and was sponsored by Given Imaging, Inc (Yoqneam, Israel).

\section{Materials and Methods}

\section{Equipment and Study End Points}

The PillCam COLON 2 (Given Imaging Inc, Yoqneam, Israel) capsule was used in this study. The PillCam COLON 2 system includes the second-generation ingestible capsule, sensors attached to the abdomen that receive capsule images, a data recorder, and a software package that displays the images on a workstation and can create procedure reports.

The primary end point of this prospective study was the accuracy (sensitivity and specificity) of the PillCam COLON 2 for detecting patients with polyps $6 \mathrm{~mm}$ or larger compared with conventional colonoscopy. All study subjects were asymptomatic candidates for colorectal cancer screening.

Secondary end points were the accuracy of the PillCam COLON 2 for the detection of patients with polyps $10 \mathrm{~mm}$ or larger in size, and the incidence of adverse events with the PillCam COLON 2.

\section{Design Overview}

The study NCT number is 01372878 . There were 10 US centers ( 2 academic, 8 private practice) and 6 (all academic) in Israel. The study was approved by the Institutional Review Boards at each center. Recruitment began in June 2011 and ended in April 2012. Patients were recruited from colonoscopy schedules (not consecutively) that listed their indication as screening. After obtaining informed consent, patients underwent the PillCam COLON 2 procedure. Capsule images were interpreted by 1 of 5 central readers who documented polyp location and measured size with a software tool. Four to 6 weeks later (after the capsule report was completed), the subjects underwent a colonoscopy. The colonoscopist was blinded to the capsule findings. During colonoscopy each polyp was photographed with an open 9-mm biopsy forceps aligned on its longest axis. The polyp then was resected and sent to pathology. Polyp location was estimated using endoscopic criteria. After colonoscope withdrawal from the subject, the capsule findings were unblinded. If the capsule had detected a polyp $6 \mathrm{~mm}$ or larger in size that was not seen by colonoscopy, the colonoscopist immediately repeated the colonoscopy.

\section{Inclusion and Exclusion Criteria}

Subjects were ages 50-75 years and were classified as average risk. ${ }^{4}$ Subjects were excluded for the following reasons: a history of colorectal cancer (CRC) or polyp; any colon imaging study (colonoscopy, barium enema, or computed tomography [CT] colonography) in the previous 5 years; first-degree relative with CRC at age younger than 60 years; 2 or more first-degree relatives with CRC at any age; familial adenomatous polyposis, Lynch syndrome, or other genetic syndrome with increased CRC risk; inflammatory bowel disease; hematochezia; melena; iron deficiency with or without anemia; positive fecal blood test; known or suspected bowel obstruction; congestive heart failure; diabetes; abdominal surgery in the past 6 months unless it was considered unlikely to cause bowel obstruction; any implanted electromedical device; allergy or other known contraindication to study medicines; anticipated magnetic resonance imaging within 1 week of capsule ingestion; risk factors for capsule retention (eg, intestinal tumors, strictures, and so forth); active fistulas; chronic constipation; motility disorders; delayed gastric emptying; clinically significant renal disease; pregnant or nursing; of childbearing age and not practicing contraception; any condition deemed life-threatening by the investigator; or current participation in another trial testing an investigational drug or device.

\section{PillCam COLON 2 Procedure}

The capsule preparation consisted of 4 senna tablets (12-mg senna) at bedtime 2 days before the procedure, clear liquids the day before the procedure, $2 \mathrm{~L}$ of sulfate-free polyethylene glycol electrolyte lavage solution (PEG-ELS; Nulytely; Braintree Laboratories, Braintree, MA) between 7 and 9 PM the evening before ingestion, and $2 \mathrm{~L}$ the morning of ingestion approximately 45 minutes before capsule ingestion. After capsule ingestion at the endoscopy center, the capsule position was monitored in real time using the data recorder image display. ${ }^{5}$ After the capsule exited the stomach, the patient ingested 6 oz of oral sulfate solution (SUPREP; Braintree Laboratories) diluted to $16 \mathrm{oz}$ with water, followed by $1 \mathrm{~L}$ of water over the next hour. The oral sulfate solution is the first boost and propels the capsule through the small intestine and adds fluid to the colon. The investigator had the option of administering $10 \mathrm{mg}$ metoclopramide orally for gastric capsule retention more than 1 hour. If the capsule was not excreted by 3 hours after ingestion of the first boost, the patient was given a second boost $(3 \mathrm{oz}$ oral sulfate solution diluted in water to $8 \mathrm{oz}$ followed by $1 \mathrm{~L}$ of water over the next hour). If the capsule was not excreted by 2 hours after the second boost the patient self-administered a 10-mg bisacodyl suppository. A standard full meal was allowed after capsule excretion or beginning 2 hours after the suppository. Subjects kept a timed diary of activity and key procedure steps including capsule excretion. Subjects could leave the unit 10 hours after capsule ingestion if the capsule was not yet excreted. Subjects who left before excretion were instructed to disconnect the recorder at excretion or 12 hours after ingestion (whichever came first).

\section{Capsule Video Reading}

Images were downloaded, sent to the sponsor within 3 days, and forwarded to a central reader. Each central reader 
was an experienced colonoscopist but not a site investigator, and received structured training in reading colon capsule videos. Each video was read within 3 weeks of receipt (maximum, 5 videos/wk/reader). For each video the reader recorded the preparation (excellent, good, fair, or poor using a validated system ${ }^{6}$ ) in 5 segments (cecum, ascending, transverse, descending-sigmoid, and rectum) and an overall preparation score. Location was estimated using landmarks visible in the video (particularly the cecum and anus), and a program that displays the approximate position of the capsule in the abdominal-pelvic cavity. This software is activated by marking the times the capsule reaches the cecum, hepatic and splenic flexures, and exit from the anus. Polyp size was estimated using Polyp Size Estimation software (Given Imaging Inc). In brief, the reader placed a cursor at one end of the longest visible dimension of the polyp and then moved the cursor to the other end of that dimension, and the software calculated the length. Findings were saved as photographs with detailed descriptions and delivered to the sponsor within 2 weeks.

\section{Follow-up Evaluation of the Capsule Procedure}

Subjects were contacted 5-9 days after the capsule procedure to evaluate changes in their well-being. If needed, a radiograph was ordered to verify capsule excretion.

\section{Colonoscopy Procedure}

Colonoscopy, blinded to the capsule findings, was performed 4-6 weeks after the capsule procedure. Bowel preparation was as follows: 4 senna tablets $(12 \mathrm{mg})$ at bedtime 2 days before, clear liquids the day before, 2 L Nulytely between 7 and 9 PM the evening before, and 2 L Nulytely on the morning of the colonoscopy. Cleansing was recorded for each colorectal segment.

All 49 study colonoscopists were trained in polyp measurement using an open 9-mm forceps (Radial Jaw 4; Boston Scientific, Natick, MA). A web program was used to train and test investigators in polyp size measurement before study initiation. ${ }^{7}$ Sedation was the usual practice at the respective sites. Each detected polyp was measured in vivo by opening the forceps and twisting the sheath until the long 9-mm axis of the open cups was aligned with the longest axis of the polyp. The forceps then were pushed against the polyp and photographed. Each photograph was submitted to the study sponsor. Polyps were removed using forceps or snares at the colonoscopist's discretion. Histologic assessment was performed at the respective study centers. A withdrawal time of 6 minutes or more was required for all colonoscopies, and all colonoscopies were videorecorded. After the colonoscopy was completed, capsule findings were removed from a sealed envelope and shown to the colonoscopist. If the findings included a polyp 6 mm or larger and the colonoscopy did not show a matched polyp (see later), the colonoscopist immediately re-inserted the colonoscope, verified whether the polyp was present, and removed it if identified.

Polyps $6 \mathrm{~mm}$ or larger detected by capsule and matched by the blinded or unblinded colonoscopies were considered truepositive capsule detections. Polyps detected by capsule that were $6 \mathrm{~mm}$ or larger and not matched by either the blinded or unblinded colonoscopy were considered false-positive capsule detections.

\section{Follow-up Evaluation of the Colonoscopy Procedure}

Subjects were contacted 5-9 days after the colonoscopy to evaluate any changes in their well-being.

\section{Polyp Matching}

In the primary analysis, polyps were considered a match if the size measured by the capsule and its $50 \%$ plus- $50 \%$ minus range overlapped with the $50 \%$ plus- $50 \%$ minus range of the size measured by the colonoscopy, and the location estimates by the 2 methods were in the same or adjacent segments. Thus, if a polyp measured $10 \mathrm{~mm}$ by capsule, the $50 \%$ plus-minus range would be 5-15 $\mathrm{mm}$. If a polyp in the same or an adjacent segment by conventional colonoscopy was $6 \mathrm{~mm}(50 \%$ plus-minus range, $3-9 \mathrm{~mm}$ ) then the 2 polyps are a potential match because the $50 \%$ plus-minus ranges overlap. The largest size determined by the 2 methods was used to assign the polyp to the following groups: $5 \mathrm{~mm}$ or less, $6 \mathrm{~mm}$ or larger, and/or $10 \mathrm{~mm}$ or larger in size.

In a separate analysis, polyps were matched by the same size methods but without reference to the colonic segment (ie, a polyp localized in any segment by one technique could match a polyp in any segment by the other technique).

\section{Pathology}

Lesions interpreted as tubular, tubulovillous, or villous adenomas are referred to herein as conventional adenomas. Lesions interpreted as hyperplastic polyps, sessile serrated polyps, sessile serrated adenoma, serrated adenoma, or traditional serrated adenoma are referred to as belonging to the serrated class. Interpretation of sessile serrated adenoma or serrated adenoma were reclassified as sessile serrated polyp because the World Health Organization considers sessile serrated polyp and sessile serrated adenoma synonymous. ${ }^{8}$ There was no central review of pathology and no training of the site pathologists.

\section{Safety}

Patients were queried at each visit and by telephone 5-9 days after each procedure for adverse events. A serious adverse event was one that resulted in death, serious deterioration in health, permanent injury to a body structure or function, hospitalization, a medical or surgical intervention that was needed to prevent a life-threatening injury or illness, or that led to fetal distress, fetal death, or a birth defect.

\section{Sample Size}

The sample size was based on estimating capsule sensitivity for detecting patients with polyps $6 \mathrm{~mm}$ or larger in size of at least $80 \%$ with precision of $10 \%$. The prevalence of polyps $6 \mathrm{~mm}$ or larger in a screening population was assumed to be $20 \%$ or higher. Assuming a type I error $(\alpha)$ of $5 \%$ and power of $80 \%$, a sample size of 628 subjects was needed. For the secondary end point of detecting patients with polyps $10 \mathrm{~mm}$ or larger in size, and assuming sensitivity of at least $80 \%$, precision of $10 \%$, and prevalence of $5 \%$ of lesions of this size, and an $\alpha$ value of $5 \%$, a sample of 690 subjects was needed. Assuming a drop-out rate of $15 \%$, a sample size of 794 subjects was planned. 


\section{Exclusions for the Combination of Rapid Transit and Poor Bowel Preparation}

The high rate of rapid transit through the colon was unexpected and may have been the result of the oral sulfate solution boost. Oral sulfate solution was tested as a boost before the study because the previous boost (sodium phosphate) rarely causes kidney damage. ${ }^{9}$ Although oral sulfate solution appeared useful in preliminary testing, the study results suggest it sometimes moved the capsule too quickly. The decision to exclude patients based on the combination of poor preparation and rapid transit (both were required for exclusion) was made after enrollment and completion of the study procedures.

\section{Statistical Analysis}

Sensitivity and specificity were evaluated for detection of subjects with polyps $6 \mathrm{~mm}$ or larger, or $10 \mathrm{~mm}$ or larger by constructing a $2 \times 2$ matrix (ie, capsule performance vs conventional colonoscopy), and are presented with the $95 \%$ confidence interval (CI).

The accuracy events were generated by the larger polyp size determined by the 2 imaging modalities identified in a given patient.

The sensitivity and specificity were evaluated using the unblinded results of colonoscopy. Summary statistics (median) for continuous variables were calculated for the overall and per-segment transit times. Capsule and colonoscopy rates of good and excellent cleansing (ie, adequate preparation) were summarized with the $95 \%$ CI based on the binomial distribution.

All authors had access to the study data and reviewed and approved the final manuscript.

\section{Results}

\section{Exclusions}

Figure 1 diagrams the initial enrollment cohort to the final analysis cohort. Of 884 asymptomatic subjects enrolled, 695 were included in the analysis of all polyps. Six patients did not have a pathology report, therefore 689 were included in the adenoma analysis. The mean age of the 689 subjects was 57 years, and $56 \%$ were female.

There were 77 subjects (9\%) excluded for both inadequate cleansing and whole-colon transit time fewer than 40 minutes. Forty-five subjects dropped out before the capsule procedure, 15 subjects dropped out after the capsule procedure and before the colonoscopy, 15 subjects were dropped because a site was terminated early for repeated protocol noncompliance, in 12 subjects the capsule did not reach the colon by 12 hours after ingestion, 10 subjects had protocol violations during the colonoscopy, in 8 subjects the capsule did not leave the cecum, 5 subjects had moderate adverse events before the capsule (related to bowel preparation) and did not ingest the capsule, and 2 subjects could not ingest the capsule.

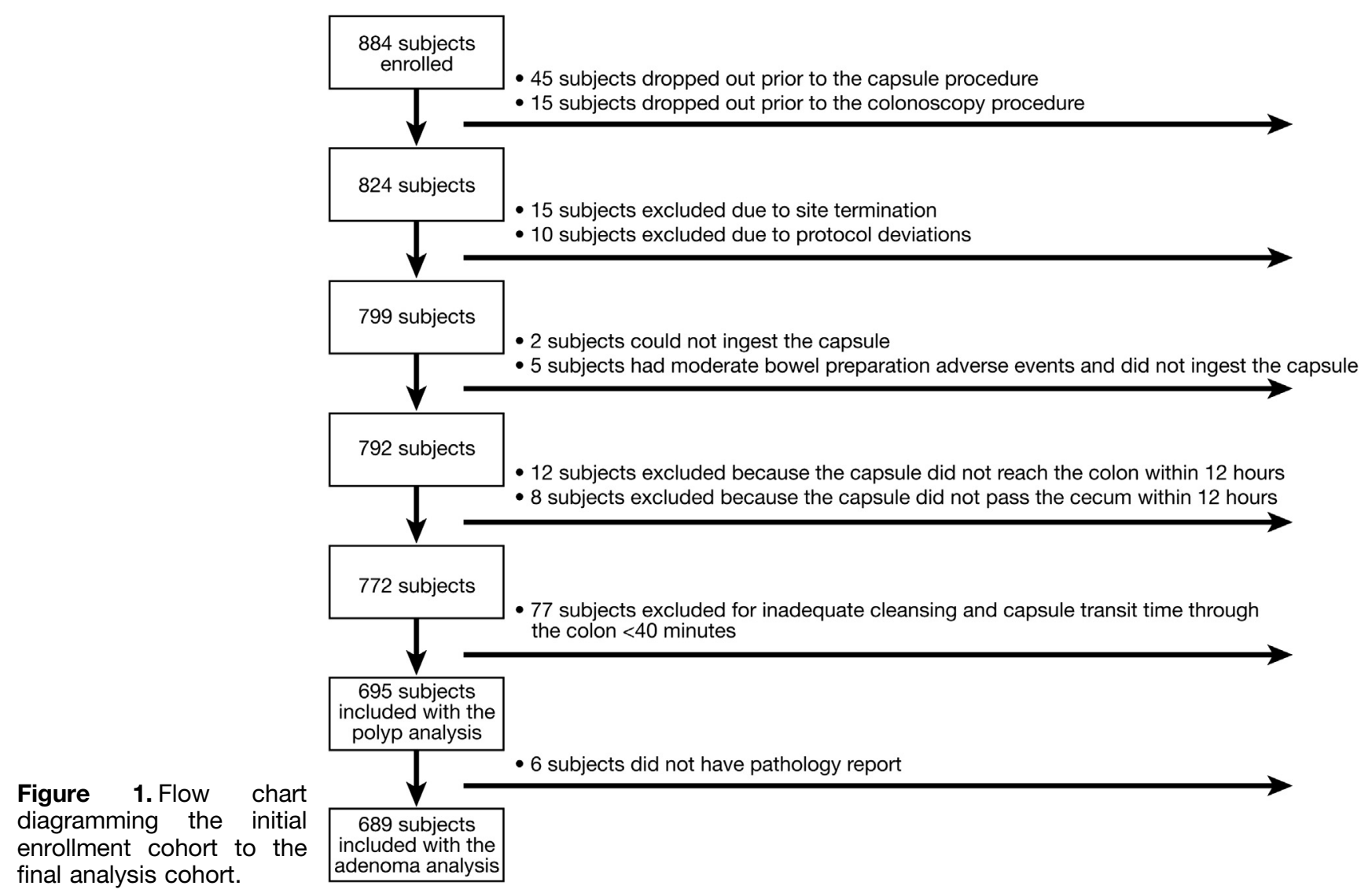




\section{Procedures}

Bowel preparation was adequate (excellent or good) for the entire colon in $80 \%(95 \% \mathrm{CI}, 76 \%-83 \%)$ of capsule procedures. The cecal cleansing level was adequate in $67 \%$ (95\% CI, 63\%-70\%), ascending colon cleansing level was adequate in $74 \%$ (95\% CI, 71\%-78\%), transverse colon cleansing level was adequate in $77 \%$ (95\% CI, 73\%-80\%), descending-sigmoid colon cleansing level was adequate in $82 \%$ (95\% CI, 79\%-85\%), and rectum cleansing level was adequate in $65 \%$ (95\% CI, 61\%-69\%). Among 689 subjects evaluated for conventional adenoma performance, 522 (76\%; 95\% CI, 72\%-79\%) excreted the capsule within 6 hours after ingestion, 607 (88\%; 95\% CI, 85\%-90\%) excreted the capsule within 8 hours, 626 (91\%; 95\% CI, 88\%-923\%) within 10 hours, and 636 (92\%; 95\% CI, 90\%94\%) excreted the capsule within 12 hours. The median transit time of the capsule through the colon before and after all exclusions was 1 hour 16 minutes and 1 hour 39 minutes, respectively (Supplementary Table 1). The total transit time through the colon was shorter than transit times in previous trials of capsule colonoscopy (Supplementary Table 1).

Colonoscopy was complete to the cecum in $99.6 \%$ (95\% CI, 99\%-100\%) of subjects. Bowel preparation was adequate (excellent or good) in $93 \%$ of subjects $(95 \% \mathrm{CI}$, $91 \%-95 \%)$. The cecal cleansing level was adequate in $88 \%$ (95\% CI, 85\%-90\%), ascending colon cleansing level was adequate in $91 \%$ (95\% CI, 89\%-93\%), transverse colon cleansing level was adequate in $92 \%$ (95\% CI, 90\%-94\%), descending-sigmoid colon cleansing level was adequate in 92\% (95\% CI, 90\%-94\%), and rectum cleansing level was adequate in $93 \%$ (95\% CI, 91\%-95\%). The mean withdrawal time in subjects with no lesions detected was 10.6 minutes (SD, $4.2 \mathrm{~min}$; range, 5-32 min). The collective adenoma detection rate (ADR) of colonoscopists for the study was $39 \%$. Among 17 colonoscopists who performed 10 or more colonoscopies, the range of ADRs was $8 \%-63 \%$, and 3 colonoscopists had ADRs below the recommended $20 \%$ threshold. ${ }^{10,11}$ The size of all polyps and the histology (when available) detected at colonoscopy is shown in Table 1. Of 340 lesions detected and retrieved for histology by colonoscopy that were $6 \mathrm{~mm}$ or larger in size (excluding the 4 cancers), 211 (62\%) were conventional adenomas and $129(38 \%)$ were in the serrated class.

Table 1.Pathology Report of All Polyps Detected by Conventional Colonoscopy

\begin{tabular}{lccc}
\hline & \multicolumn{3}{c}{ Conventional colonoscopy } \\
\cline { 2 - 4 } \multicolumn{1}{c}{ Polyp pathology } & $\leq 5 \mathrm{~mm}$ & $6-9 \mathrm{~mm}$ & $\geq 10 \mathrm{~mm}$ \\
\hline Cancer & - & - & 4 \\
Conventional adenoma & 439 & 142 & 69 \\
Sessile serrated polyp & 14 & 17 & 17 \\
Hyperplastic polyp & 458 & 76 & 19 \\
Biopsy specimen not taken & 192 & 19 & 5 \\
$\quad$ or not retrieved & & & \\
Total & 1103 & 254 & 114 \\
& & & \\
\hline
\end{tabular}

Table 2. Location of Lesions $6 \mathrm{~mm}$ or Larger and $10 \mathrm{~mm}$ or Larger Detected by Capsule Colonoscopy and Conventional Colonoscopy

\begin{tabular}{|c|c|c|c|c|}
\hline & \multicolumn{2}{|c|}{ Capsule } & \multicolumn{2}{|c|}{ Conventional colonoscopy } \\
\hline & $\geq 6 \mathrm{~mm}$ & $\geq 10 \mathrm{~mm}$ & $\geq 6 \mathrm{~mm}$ & $\geq 10 \mathrm{~mm}$ \\
\hline Cecum & 46 & 15 & 40 & 15 \\
\hline Ascending & 98 & 32 & 97 & 39 \\
\hline Transverse & 44 & 12 & 84 & 23 \\
\hline $\begin{array}{l}\text { Descending- } \\
\text { sigmoid }\end{array}$ & 126 & 28 & 112 & 32 \\
\hline Rectum & 78 & 24 & 35 & 5 \\
\hline Total & 392 & 111 & 368 & 114 \\
\hline
\end{tabular}

\section{Capsule Performance}

Capsule identified 392 lesions $6 \mathrm{~mm}$ or larger and 111 lesions $10 \mathrm{~mm}$ or larger in size. Colonoscopy detected 368 lesions $6 \mathrm{~mm}$ or larger and 114 lesions $10 \mathrm{~mm}$ or larger (Table 2).

Capsule detection of patients with polyps and with conventional adenomas $6 \mathrm{~mm}$ or larger and $10 \mathrm{~mm}$ or larger is shown in Table 3. The capsule sensitivities for detecting subjects with any polyp $6 \mathrm{~mm}$ or larger and $10 \mathrm{~mm}$ or larger were $81 \%$ (95\% CI, 77\%-84\%) and $80 \%$ (95\% CI, $74 \%-86 \%$ ), respectively (per-segment matching), and the specificities were 93\% (95\% CI, 91\%-95\%) and 97\% (95\% CI, 96\%-98\%), respectively.

For the analysis of capsule detection of patients with conventional adenomas, lesions in the serrated class were ignored, except that a lesion detected by capsule that proved to not be a conventional adenoma was considered a falsepositive capsule detection. Capsule sensitivities for detecting subjects with conventional adenomas $6 \mathrm{~mm}$ or larger and $10 \mathrm{~mm}$ or larger were $88 \%(95 \% \mathrm{CI}, 82 \%-93 \%)$ and $92 \%(95 \% \mathrm{CI}, 82 \%-97 \%)$, respectively, and the specificities were $82 \%(95 \% \mathrm{CI}, 80 \%-83 \%)$ and $95 \%$ (95\% CI, 94\%95\%), respectively (Table 3).

Figure 2 summarizes the capsule accuracy for detecting patients with at least 1 polyp as well as 1 conventional adenoma $6 \mathrm{~mm}$ or larger and $10 \mathrm{~mm}$ or larger in size with matching lesions by segment analysis.

Supplementary Table 2 shows the capsule accuracy in the right vs left colon for patients with polyps and conventional adenomas $6 \mathrm{~mm}$ or larger and $10 \mathrm{~mm}$ or larger in size. The sensitivity of the capsule in the right colon for patients with any polyp $6 \mathrm{~mm}$ or larger was $72 \%$ (95\% CI, 65\%-79\%), which was less than the left colon sensitivity of 88\% (95\% CI, 82\%-93\%). There were no other significant differences in right vs left capsule performance.

There were no significant differences in the sensitivity or specificity between capsule readers except for one reader who had lower specificity for lesions $6 \mathrm{~mm}$ or larger (Supplementary Table 3).

\section{Detection of Lesions by Histologic Class}

Table 4 provides additional detail regarding per-patient sensitivity of the capsule by histologic class. The sensitivity 
Table 3. Accuracy of PillCam COLON 2 for Detecting Patients With at Least One Polyp With Size 6 mm or Larger and $10 \mathrm{~mm}$ or Larger and at Least One Conventional Adenoma $6 \mathrm{~mm}$ or Larger and $10 \mathrm{~mm}$ or Larger in Size With Matching Lesions by Segment Analysis and by Entire Colon Analysis

\begin{tabular}{|c|c|c|c|c|c|}
\hline \multirow[b]{2}{*}{ Lesion size, $\mathrm{mm}$} & \multirow[b]{2}{*}{$\begin{array}{c}\text { Colonoscopy } \\
\text { prevalence, n (\%) }\end{array}$} & \multicolumn{2}{|c|}{ Capsule per segment analysis } & \multicolumn{2}{|c|}{ Capsule per entire colon analysis } \\
\hline & & $\begin{array}{l}\text { Sensitivity, \% } \\
\text { (95\% Cl) }\end{array}$ & $\begin{array}{l}\text { Specificity, \% } \\
\text { (95\% Cl) }\end{array}$ & $\begin{array}{l}\text { Sensitivity, \% } \\
\text { (95\% Cl) }\end{array}$ & $\begin{array}{l}\text { Specificity, \% } \\
\text { (95\% Cl) }\end{array}$ \\
\hline \multicolumn{6}{|l|}{ Any polyp } \\
\hline$\geq 6$ & $192(28 \%)$ & $81(77-84)$ & $93(91-95)$ & $87(82-90)$ & $94(92-96)$ \\
\hline$\geq 10$ & $79(11 \%)$ & $80(74-86)$ & 97 (96-98) & $85(77-92)$ & 97 (96-99) \\
\hline \multicolumn{6}{|c|}{ Any conventional adenoma } \\
\hline$\geq 6$ & $107(16 \%)$ & $88(82-93)$ & $82(80-83)$ & $91(85-95)$ & $83(80-86)$ \\
\hline$\geq 10$ & $43(6 \%)$ & $92(82-97)$ & 95 (94-95) & $92(82-97)$ & $95(93-97)$ \\
\hline
\end{tabular}

for conventional adenomas first was calculated without reference to lesions in the serrated class (Table 4, top row). Next, the sensitivity of the capsule for detecting patients with a sessile serrated polyp $6 \mathrm{~mm}$ or larger and $10 \mathrm{~mm}$ or larger, but no conventional adenoma $6 \mathrm{~mm}$ or larger, was calculated without reference to patients with hyperplastic polyps (Table 4, row 2). Finally, patients with hyperplastic polyps $6 \mathrm{~mm}$ or larger but no conventional adenomas or sessile serrated polyps $6 \mathrm{~mm}$ or larger were considered (Table 4, row 3). Sessile serrated polyps accounted for $26 \%$ of false-negative capsule detections of patients with polyps $6 \mathrm{~mm}$ or larger, and hyperplastic polyps accounted for $37 \%$ of this group.

\section{Detection With Entire Colon Matching}

In a separate analysis we calculated the capsule performance for detecting patients with polyps and conventional adenomas $6 \mathrm{~mm}$ or larger and $10 \mathrm{~mm}$ or larger in size without requiring that polyps be matched by segment (Table 3). Sensitivity was numerically, but not significantly, higher with entire-colon compared with per-segment matching.

\section{Contribution of Unblinding}

There were 52 cases in which a lesion $6 \mathrm{~mm}$ or larger was detected by capsule and not by conventional colonoscopy, including 24 cases in which a lesion $10 \mathrm{~mm}$ or larger was detected by capsule. Among the 52 cases, repeat colonoscopy identified a matching lesion in 22 cases, including 7 of 24 cases with a polyp $10 \mathrm{~mm}$ or larger detected by the capsule and not by conventional colonoscopy. These cases were designated true-positive capsule detections. Figure 3 shows examples of $6 \mathrm{~mm}$ or larger lesions shown by the capsule that were not verified by colonoscopy and were designated false-positive capsule detections.

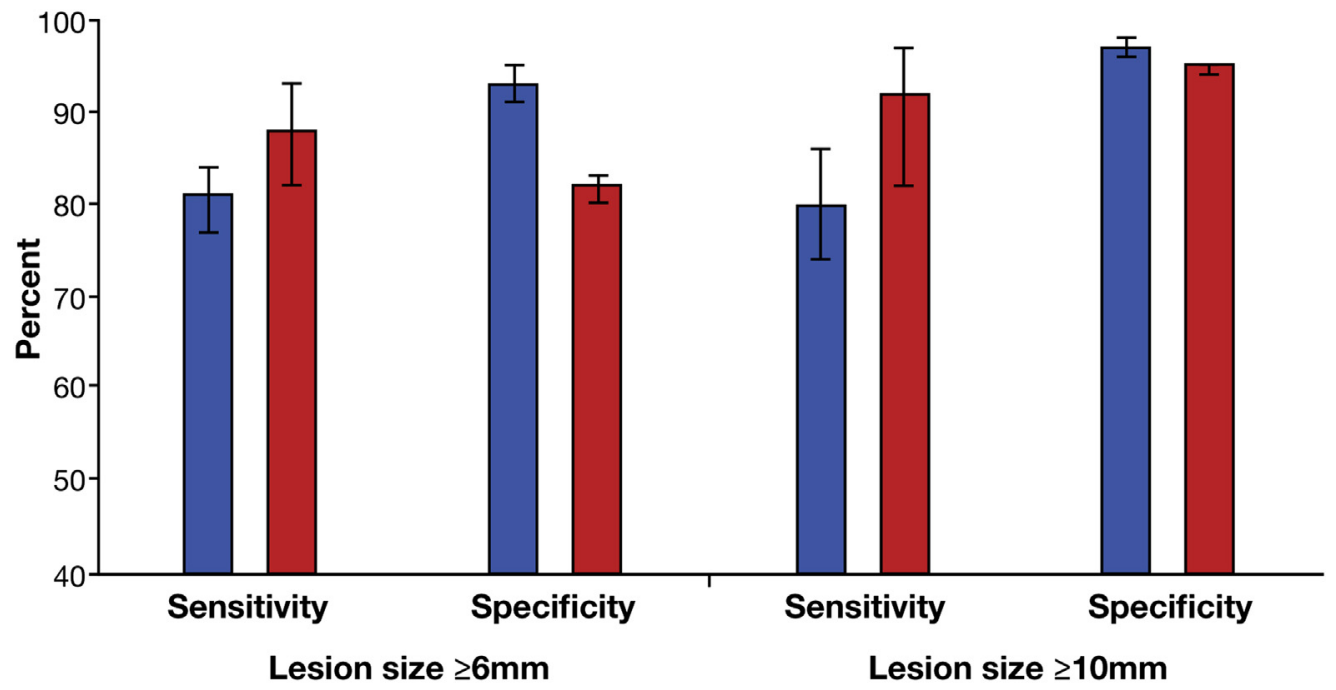

Polyp analysis $\square$ Conventional adenoma analysis

Figure 2. Summary of capsule accuracy for detecting patients with at least 1 polyp (blue) and at least 1 conventional adenoma (red) $6 \mathrm{~mm}$ or larger and $10 \mathrm{~mm}$ or larger in size with matching lesions by segment. The error bars indicate $95 \% \mathrm{Cls}$. The specificity for adenomas $6 \mathrm{~mm}$ or larger is lower than the specificity for adenomas $10 \mathrm{~mm}$ or larger, probably because nonadenomatous polyps detected by the capsule were considered as capsule false positives for the adenoma analysis, and a higher proportion of $10 \mathrm{~mm}$ or larger polyps are adenomas. 
Table 4. Sensitivity of Capsule for Detection of Patients (PerSegment Analysis) With Colorectal Lesions of Different Histology

\begin{tabular}{lll}
\hline & \multicolumn{2}{c}{ Capsule sensitivity, \% (95\% Cl) } \\
\cline { 2 - 3 } Polyp pathology & $\geq 6 \mathrm{~mm}$ & $\geq 10 \mathrm{~mm}$ \\
\hline Adenoma & $88(82-93)$ & $92(82-97)$ \\
Sessile serrated polyp & $29(10-56)$ & $33(10-65)$ \\
Hyperplastic polyp & $74(62-84)$ & $81(58-95)$
\end{tabular}

NOTE. See text for details of analysis.

\section{Cancers}

Conventional colonoscopy detected 4 cancers in 4 patients. The cancers ranged in size from 10 to $80 \mathrm{~mm}$. The capsule identified 3 cancers for a per-lesion sensitivity of $75 \%$. The cancer that was missed was a $10-\mathrm{mm}$ sessile lesion in the sigmoid colon. Unblinded review of the capsule video determined that the cancer was photographed by the capsule in multiple frames. The same patient had 2 cecal lesions (an 11-mm tubular adenoma and a 7-mm hyperplastic polyp) identified by the capsule.
Therefore, the per-patient sensitivity of capsule for the 4 cancers was $100 \%$.

\section{Performance in Excluded Patients}

In the 77 patients excluded for both inadequate preparation and a colon transit time of fewer than 40 minutes, capsule sensitivities for detecting patients with any polyp $6 \mathrm{~mm}$ or larger and $10 \mathrm{~mm}$ or larger were $68 \%$ and $71 \%$, respectively (polyps matched by segment). The capsule sensitivities for detecting patients with any conventional adenoma $6 \mathrm{~mm}$ or larger and $10 \mathrm{~mm}$ or larger were $70 \%$ and $73 \%$, respectively.

\section{Adverse Events}

There were no serious adverse events associated with the capsule. One patient was hospitalized for 1 day after colonoscopy with abdominal pain.

There were 142 nonserious adverse events in $101 \mathrm{pa}-$ tients (11\% of the 884 enrolled patients), with 128 related to bowel preparation including vomiting, nausea, headache, dizziness, gagging, diarrhea, hemorrhoidal bleeding, bloating, rash, abdominal pain, syncope, tinnitus, chest burning, chills, and flu-like symptoms, which resolved in all cases
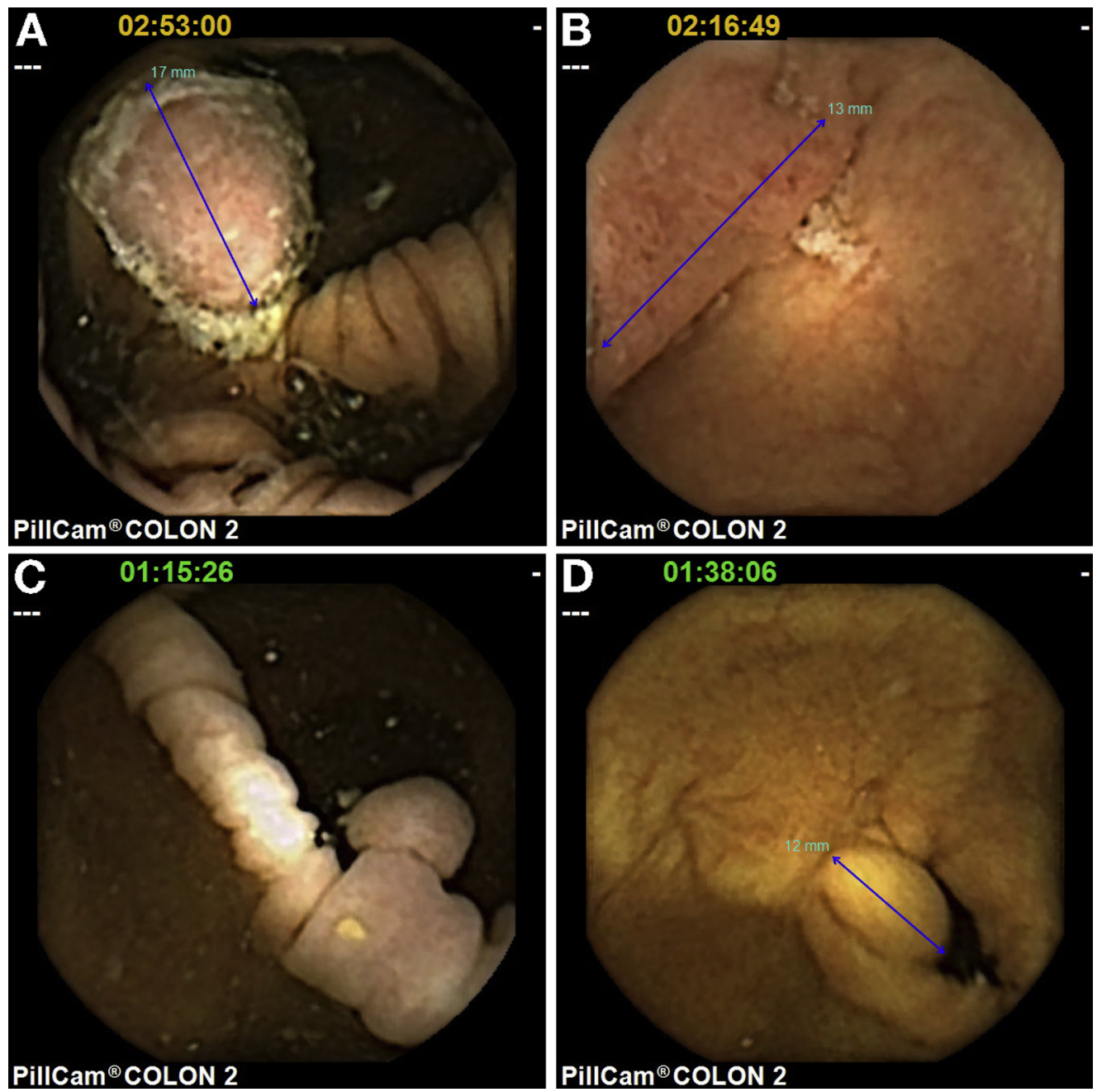

Figure 3. $(A-D)$ Capsule photographs of polyps that were not verified by either the blinded or unblinded colonoscopy. 
within 1 month and in $92 \%$ within 1 day. Eleven (8\%) of the 142 nonserious adverse events were related to colonoscopy and included fever, headache, abdominal pain, bloating, and rectal bleeding. All resolved within 8 days. Three nonserious adverse events were related to the capsule procedure included gagging, vomiting, and abdominal cramping, and each resolved on the same day.

\section{Discussion}

This report describes a capsule endoscopy study for colorectal polyp detection in asymptomatic persons.

Several aspects of the study design and the results of this trial are important to consider because they indicate that a complete understanding of capsule performance relative to colonoscopy is difficult to achieve. First, polyps were matched by colon segment whereas in previous trials of the second-generation capsule polyps were matched by the entire colon. ${ }^{2,3}$ When we performed a secondary analysis of our results with matching by the entire colon, the results for capsule sensitivity were numerically better and comparable with those of the initial smaller studies. ${ }^{2,3}$ Both colonoscopy and capsule are inferior for localization compared with CT colonography (CTC). Inaccurate localization by one or both tests in this study could have reduced the sensitivity of the capsule. Because a capsule finding of a polyp $6 \mathrm{~mm}$ or larger could be expected to generate a follow-up complete colonoscopy in clinical practice, whole-colon matching of polyp findings between the capsule and colonoscopy in clinical trials seems clinically relevant. Second, matching polyps by size, even with prespecified rules for polyp measurement and seemingly liberal rules for matching, is subject to error. In a preliminary study of the accuracy of colonoscopy at measuring polyps, we found that colonoscopists presented with the same photographs of polyps with aligned forceps reached variable conclusions about polyp size. ${ }^{7}$ Anecdotally, analysis of video recordings from this study suggested a variety of other errors occur in size measurement during colonoscopy, such as failure to expose the longest polyp dimension for photography, failure to push the forceps against the polyp, failure to orient the forceps along the longest axis of the polyp, and so forth. In addition, it might be expected that the capsule and colonoscopy would photograph the same polyp from different perspectives, with different degrees of luminal distention, and with different magnifications (capsule photographs are through water and colonoscopy photographs are through gas). Furthermore, the software used to measure polyp size during the capsule studies had a $40 \%$ plus-minus error range when tested on balls of known size in a model (Given Imaging, Inc, unpublished data), but there are no published studies of the accuracy of this software. With regard to these issues in size matching, we used a size matching rule in this study (polyps matched if the 50\% plus range of the smaller size measurement was within the $50 \%$ minus range of the larger size measurement, and either the colonoscopy size or the capsule size measurement could serve as the larger measure) that was more liberal (allowed more frequent polyp matching) than that used in previous studies. ${ }^{2,3}$ Thus, in this study we used a more liberal size matching rule and a stricter location matching rule compared with previous studies. ${ }^{2,3}$ Any set of matching rules for polyps detected by the capsule and colonoscopy might operate to increase or decrease the calculated sensitivity of capsule incorrectly. Third, colonoscopy is known to be an imperfect gold standard. ${ }^{12-14}$ In this study, the average performance of colonoscopy was excellent (overall ADR, 39\%), but 3 colonoscopists had ADRs that were below the recommended thresholds, and in some instances a polyp clearly visible by capsule was not verified by colonoscopy. In these cases a polyp that should be a true positive for the capsule was counted as false. Finally, any imaging test measured against colonoscopy will have its apparent performance affected by the quality of the colonoscopy. For example, the best results ever reported for the performance of CTC came from a center where the combined ADR of the colonoscopists during the study was only $16.5 \%{ }^{15}$ In that case, the sensitivity of CTC likely was improved by the relatively poor performance of the gold standard colonoscopies. In previous studies of the capsule, the average ADR of the colonoscopies was not reported. ${ }^{1-3}$ The average ADR in this study was among the highest ever reported for a mixed-gender screening population. ${ }^{14-22}$ We suggest that ADRs be reported in future studies of noncolonoscopic imaging because this information allows assessment of the colonoscopy quality as the gold standard in the study, and may allow improved understanding of how varying results for performance of the test modality occur in different studies.

Lesions in the serrated class were not detected well by the capsule in this study compared with conventional adenomas. In previous studies of the capsule, ${ }^{1-3}$ detection of serrated lesions was not reported. Sessile serrated polyps are commonly flat or sessile, pale in color, and subtle in appearance when viewed by colonoscopy. ${ }^{23}$ Initial studies suggest colonoscopists have more variability in the detection of sessile serrated polyps compared with conventional adenomas. $^{24,25}$ CTC also has difficulty detecting these lesions, ${ }^{26}$ and a recent trial found that fecal DNA testing had some sensitivity for serrated lesions whereas fecal immunochemical testing had no sensitivity. ${ }^{16}$ Our results indicate that additional work is needed to understand the appearance of serrated lesions at capsule and to improve their detection.

The sensitivity of CTC for patients with adenomas $6 \mathrm{~mm}$ or larger in the National CT Colonography trial was $78 \%{ }^{27}$ which is comparable with the $88 \%$ sensitivity found in the current trial. Given that both CTC and the capsule are intended for detection of lesions $6 \mathrm{~mm}$ or larger and not diminutive lesions, and that CTC is recommended at 5-year intervals when negative, ${ }^{28,29}$ our results suggest that the capsule also might be performed at 5-year intervals when used for screening.

The specificities achieved in the current study were higher than 2 previous studies of the second-generation colon capsule. ${ }^{2,3}$ This finding may reflect the more liberal size matching rule used in the current study, the training of the central readers, or the unblinding after colonoscopy, which converted some cases from false to true positives. 
The most significant limitation of this trial was that we excluded 77 patients for the combination of short transit time and poor preparation and this exclusion was not planned before the study. The short transit times likely were related to use of oral sulfate solution as a boost. Outside the United States, sodium phosphate frequently is used as the boost, but because of the occurrence of rare cases of acute irreversible nephropathy after sodium phosphate, the continued use of this agent was considered inappropriate in the United States. Exclusion of these patients is consistent with the anticipated labeling of the capsule by the US Food and Drug Administration, which indicate that patients with a combination of poor preparation and a colonic transit time of fewer than 40 minutes had a technically inadequate study for polyp detection. We did describe the capsule performance in the 77 excluded patients.

In summary, we found that the capsule performed well for detecting asymptomatic patients with any polyp and with any conventional adenoma $6 \mathrm{~mm}$ or larger in size. In considering the capsule as a colorectal cancer screening test, it is appropriate to consider that the bowel preparation for the capsule is more extensive than that required for colonoscopy, that technical failures (short transit time plus poor preparation) occurred in $9 \%$ of patients, and that the logistics of performing same-day colonoscopy in patients with a positive capsule study are difficult. Given these considerations and the results of this study, we conclude that colonoscopy remains the gold standard for the detection of colorectal polyps. The capsule is a good test for the detection of patients with conventional adenomas $6 \mathrm{~mm}$ or larger in size and appears to be an appropriate imaging choice for patients who cannot undergo colonoscopy or had incomplete colonoscopy. Additional study of the capsule, including efforts to improve the detection of serrated lesions, appears warranted.

\section{Supplementary Material}

Note: To access the supplementary material accompanying this article, visit the online version of Gastroenterology at www.gastrojournal.org, and at http://dx.doi.org/10.1053/ j.gastro.2015.01.025.

\section{References}

1. Van Gossum A, Munoz-Navas M, Fernandez-Urien I, et al. Capsule endoscopy versus colonoscopy for the detection of polyps and cancer. N Engl J Med 2009; 361:264-270.

2. Eliakim R, Yassin K, Niv Y, et al. Prospective multicenter performance evaluation of the second-generation colon capsule compared with colonoscopy. Endoscopy 2009; 41:1026-1031.

3. Spada C, Hassan C, Munoz-Navas M, et al. Secondgeneration colon capsule endoscopy compared with colonoscopy. Gastrointest Endosc 2011;74:581-589.e1.

4. Levin B, Lieberman DA, McFarland B, et al. Screening and surveillance for the early detection of colorectal cancer and adenomatous polyps, 2008: a joint guideline from the American Cancer Society, the US Multi-Society Task Force on Colorectal Cancer, and the American College of Radiology. Gastroenterology 2008; 134:1570-1595.

5. Adler S, Hassan C, Metzger Y, et al. Accuracy of automatic detection of small-bowel mucosa by second-generation colon capsule endoscopy. Gastrointest Endosc 2012; 76:1170-1174.

6. Leighton JA, Rex DK. A grading scale to evaluate colon cleansing for the PillCam COLON capsule: a reliability study. Endoscopy 2011;43:123-127.

7. Rex DK, Rabinovitz R. Variable interpretation of polyp size by using open forceps by experienced colonoscopists. Gastrointest Endosc 2014;79:402-407.

8. Snover D, Ahnen DJ, Burt RW, et al. Serrated polyps of the colon and rectum and serrated ("hyperplastic") polyposis. In: Bozman FT, Carneiro F, Hruban RH, et al, eds. WHO classification of tumours. Pathology and genetics. Tumours of the digestive system. 4th ed. Berlin: Springer-Verlag, 2010.

9. Markowitz GS, Perazella MA. Acute phosphate nephropathy. Kidney Int 2009;76:1027-1034.

10. Rex DK, Bond JH, Winawer S, et al. Quality in the technical performance of colonoscopy and the continuous quality improvement process for colonoscopy: recommendations of the U.S. Multi-Society Task Force on Colorectal Cancer. Am J Gastroenterol 2002;97:1296-1308.

11. Rex DK, Petrini JL, Baron TH, et al. Quality indicators for colonoscopy. Gastrointest Endosc 2006;63:S16-S28.

12. Rex DK, Cutler CS, Lemmel GT, et al. Colonoscopic miss rates of adenomas determined by back-to-back colonoscopies. Gastroenterology 1997;112:24-28.

13. Rex DK. Maximizing detection of adenomas and cancers during colonoscopy. Am J Gastroenterol 2006; 101:2866-2877.

14. Corley D, Jensen CD, Marks AR, et al. Adenoma detection rate and risk of colorectal cancer and death. N Engl J Med 2014;370:1298-1306.

15. Hsu R, Gopal D, Reichelderfer M, et al. Direct comparison of an optical colonoscopy and virtual colonoscopy colorectal cancer screening program in the average risk patient. Gastrointest Endosc 2006;63:Ab82.

16. Imperiale TF, Ransohoff DF, Itzkowitz SH, et al. Multitarget stool DNA testing for colorectal-cancer screening. N Engl J Med 2014;370:1287-1297.

17. Kaminski MF, Regula J, Kraszewska E, et al. Quality indicators for colonoscopy and the risk of interval cancer. N Engl J Med 2010;362:1795-1803.

18. Rex DK, Lehman GA, Ulbright TM, et al. Colonic neoplasia in asymptomatic persons with negative fecal occult blood tests: influence of age, gender, and family history. Am J Gastroenterol 1993;88:825-831.

19. Imperiale $T$, Wagner D, Lin C, et al. Risk of advanced proximal neoplasms in asymptomatic adults according to the distal colorectal findings. N Engl J Med 2000; 343:169-174.

20. Lieberman DA, Weiss DG, Bond JH, et al. Use of colonoscopy to screen asymptomatic adults for colorectal cancer. Veterans Affairs Cooperative Study Group 380. N Engl J Med 2000;343:162-168. 
21. Church TR, Wandell M, Lofton-Day C, et al. Prospective evaluation of methylated SEPT9 in plasma for detection of asymptomatic colorectal cancer. Gut 2014;63: 317-325.

22. Barclay RL, Vicari JJ, Doughty AS, et al. Colonoscopic withdrawal times and adenoma detection during screening colonoscopy. N Engl J Med 2006;355:2533-2541.

23. Hazewinkel Y, Lopez-Ceron M, East JE, et al. Endoscopic features of sessile serrated adenomas: validation by international experts using high-resolution white-light endoscopy and narrow-band imaging. Gastrointest Endosc 2013;77:916-924.

24. Kahi CJ, Hewett DG, Norton DL, et al. Prevalence and variable detection of proximal colon serrated polyps during screening colonoscopy. Clin Gastroenterol Hepatol 2011;9:42-46.

25. Hetzel JT, Huang CS, Coukos JA, et al. Variation in the detection of serrated polyps in an average risk colorectal cancer screening cohort. Am J Gastroenterol 2010; 105:2656-2664.

26. Pickhardt PJ, Choi JR, Hwang I, et al. Nonadenomatous polyps at CT colonography: prevalence, size distribution, and detection rates. Radiology 2004; 232:784-790.

27. Johnson CD, Chen MH, Toledano AY, et al. Accuracy of CT colonography for detection of large adenomas and cancers. N Engl J Med 2008;359:1207-1217.
28. Levin B, Lieberman DA, McFarland B, et al. Screening and surveillance for the early detection of colorectal cancer and adenomatous polyps, 2008: a joint guideline from the American Cancer Society, the US Multi-Society Task Force on Colorectal Cancer, and the American College of Radiology. CA Cancer J Clin 2008;58:130-160.

29. Zalis ME, Barish MA, Choi JR, et al. CT colonography reporting and data system: a consensus proposal. Radiology 2005;236:3-9.

Received July 18, 2014. Accepted January 16, 2015.

Reprint requests

Address requests for reprints to: Douglas K. Rex, MD, 550 N. University Boulevard, Indiana University Hospital \#4100, Indianapolis, Indiana 46202 e-mail: drex@iupui.edu; fax: (317) 944-5449.

\section{Conflicts of interest}

These authors disclose the following: Douglas Rex and Samuel Adler have received consulting fees from Given Imaging, Inc; Cristina Carretero has received speaker's fees and fees for central reading from Given Imaging, Inc; Ignacio Fernandez-Urien Sainz, Wai-Keung Leung, Neofytos Papageorgiou, and Matti Waterman have received fees for central reading from Given Imaging, Inc.

Given Imaging, Inc, participated in the study design, provided funding, participated in data analysis, review, and comment on the manuscript.

The remaining authors disclose no conflicts.

\section{Funding}

Supported by Given Imaging, Inc. 
Supplementary Table 1. Median Capsule Transit Times by Segment and Whole Colon in Previous Studies of the SecondGeneration Capsule (PillCam COLON 2) and in the Current Study Before and After All Exclusions

\begin{tabular}{|c|c|c|c|c|c|}
\hline \multirow[b]{2}{*}{ Study } & \multicolumn{5}{|c|}{ Median capsule transit times in the colon } \\
\hline & Cecum & Ascending & Transverse & Left & Total colon \\
\hline Prior studies with sodium phosphate boost ${ }^{2,3}(n=265)$ & 00:06:36 & 00:03:03 & 00:01:22 & 01:14:58 & 02:07:45 \\
\hline Current study with sulfate solution boost $(n=832)$ & 00:06:07 & 00:03:18 & 00:01:22 & $00: 45: 14$ & 01:15:35 \\
\hline Current study after all exclusions ( $n=700$, after exclusions) & 00:06:25 & $00: 03: 38$ & $00: 01: 43$ & $00: 58: 30$ & $01: 39: 18$ \\
\hline
\end{tabular}

Supplementary Table 2.Sensitivity and Specificity of the Capsule in the Right Versus Left Colon

\begin{tabular}{|c|c|c|c|c|}
\hline \multirow[b]{2}{*}{ Lesion size, $\mathrm{mm}$} & \multicolumn{2}{|c|}{ Capsule: right colon } & \multicolumn{2}{|c|}{ Capsule: left colon } \\
\hline & Sensitivity, \% (95\% Cl) & Specificity, \% (95\% Cl) & Sensitivity, \% (95\% Cl) & Specificity, \% (95\% Cl) \\
\hline \multicolumn{5}{|l|}{ Any polyp } \\
\hline$\geq 6$ & $72(65-79)$ & $95(93-97)$ & $88(82-93)$ & 98 (97-99) \\
\hline$\geq 10$ & $72(60-83)$ & 98 (96-99) & $85(72-94)$ & $99(99-100)$ \\
\hline \multicolumn{5}{|c|}{$\overline{\text { Any conventional adenoma }}$} \\
\hline$\geq 6$ & $82(73-89)$ & $90(87-92)$ & $93(85-97)$ & $91(89-93)$ \\
\hline$\geq 10$ & $84(68-94)$ & $96(95-98)$ & 94 (81-99) & 98 (97-99) \\
\hline
\end{tabular}




\begin{tabular}{|c|c|c|c|c|c|c|c|c|c|c|}
\hline \multirow[b]{2}{*}{$\begin{array}{l}\text { Lesion } \\
\text { size, } m m\end{array}$} & \multicolumn{2}{|c|}{ Capsule reader 1 , videos, $\mathrm{n}^{a}$} & \multicolumn{2}{|c|}{ Capsule reader 2 , videos, $\mathrm{n}$} & \multicolumn{2}{|c|}{ Capsule reader 3 , videos, $\mathrm{n}$} & \multicolumn{2}{|c|}{ Capsule reader, 4 , videos, $\mathrm{n}$} & \multicolumn{2}{|c|}{ Capsule reader, 5 , videos, $\mathrm{n}$} \\
\hline & $\begin{array}{l}\text { Sensitivity, \% } \\
\text { (95\% Cl) }\end{array}$ & $\begin{array}{l}\text { Specificity, \% } \\
\quad(95 \% \mathrm{Cl})\end{array}$ & $\begin{array}{l}\text { Sensitivity, \% } \\
\quad(95 \% \mathrm{Cl})\end{array}$ & $\begin{array}{l}\text { Specificity, \% } \\
\text { (95\% Cl) }\end{array}$ & $\begin{array}{l}\text { Sensitivity, \% } \\
\quad(95 \% \mathrm{Cl})\end{array}$ & $\begin{array}{l}\text { Specificity, \% } \\
\text { (95\% Cl) }\end{array}$ & $\begin{array}{l}\text { Sensitivity, \% } \\
(95 \% \mathrm{Cl})\end{array}$ & $\begin{array}{l}\text { Specificity, \% } \\
\text { (95\% Cl) }\end{array}$ & $\begin{array}{l}\text { Sensitivity, \% } \\
(95 \% \mathrm{Cl})\end{array}$ & $\begin{array}{l}\text { Specificity, \% } \\
\text { (95\% Cl) }\end{array}$ \\
\hline \multicolumn{11}{|l|}{ Any polyp } \\
\hline & \multicolumn{2}{|c|}{$141^{a}$} & \multicolumn{2}{|c|}{142} & \multicolumn{2}{|c|}{130} & \multicolumn{2}{|c|}{130} & \multicolumn{2}{|c|}{152} \\
\hline$\geq 6$ & $72(59-83)$ & 96 (90-99) & 78 (64-89) & $96(90-99)$ & $76(61-87)$ & 96 (90-99) & 95 (82-99) & $98(92-100)$ & $88(77-95)$ & $80(71-88)$ \\
\hline$\geq 10$ & $64(41-82)$ & $100(97-100)$ & $85(66-96)$ & 97 (93-99) & $76(50-93)$ & $98(94-100)$ & $92(64-100)$ & $98(94-100)$ & $87(66-97)$ & $92(86-96)$ \\
\hline \multicolumn{11}{|c|}{ Any conventional adenoma } \\
\hline & \multicolumn{2}{|c|}{139} & \multicolumn{2}{|c|}{142} & \multicolumn{2}{|c|}{129} & \multicolumn{2}{|c|}{129} & \multicolumn{2}{|c|}{150} \\
\hline$\geq 6$ & $81(64-92)$ & $83(74-89)$ & $96(79-100)$ & $89(82-94)$ & $80(59-93)$ & $82(73-87)$ & $96(78-100)$ & $88(80-93)$ & $91(77-98)$ & $68(58-76)$ \\
\hline$\geq 10$ & $82(48-98)$ & 97 (92-99) & $100(82-100)$ & 95 (90-98) & $78(40-97)$ & 95 (89-98) & $90(56-100)$ & 97 (92-99) & $100(72-100)$ & $91(85-95)$ \\
\hline
\end{tabular}

${ }^{a}$ Number of videos read by each capsule reader. 